\title{
Contact dynamics of tapping mode atomic force microscopy
}

\author{
Yin Zhang*, Haisheng Zhao, Lijun Zuo \\ State Key Laboratory of Nonlinear Mechanics (LNM), Institute of Mechanics, Chinese Academy of Sciences, Beijing 100190, China
}

\section{A R T I C L E I N F O}

\section{Article history:}

Received 31 May 2012

Received in revised form

5 July 2012

Accepted 7 July 2012

Handling Editor: L.G. Tham

Available online 25 July 2012

\begin{abstract}
A B S T R A C T
A comprehensive model on the dynamics of a tilted tapping mode atomic force microscopy (AFM) is presented, which includes the multimodal analysis, mode coupling mechanisms, adhesion, contact and friction forces induced by the tilting angle. A displacement criterion of contact/impact is proposed to eliminate the assumptions of the previous models such as infinite stiffness of sample or zero impact velocity, which makes the model presented here suitable for more general AFM application scenario, especially for the soft sample case. The AFM tip mass, tip-sample damping, contact forces and intermittent contact can all induce the higher modes participation into the system motion. One degree of freedom or one mode study on the AFM contact dynamics of tapping mode is shown to be inaccurate. The Hertz and Derjaguin-Muller-Toporov models are used for the comparison study of the non-adhesive and adhesive contacts. The intermittent contact and the contact forces are the two major sources of the system nonlinearity. The rich dynamic responses of the system and its sensitivity to the initial conditions are demonstrated by presenting various subharmonic and nonperiodic motions.
\end{abstract}

(c) 2012 Elsevier Ltd. All rights reserved.

\section{Introduction}

Atomic force microscope (AFM) [1] is a powerful tool for surface characterizations. When an AFM is used to obtain the force-distance curve, the so-called "jump-to-contact" instability [2-5] often occurs. The "jump-to-contact" instability is also termed as the pull-in instability, which indicates that the structure elastic restoring force due to deformation can no longer balance the nonlinear attractive forces such as van der Waals (vdW) [6], electrostatic [7] and Casimir forces [8]. The "jump-to-contact" instability causes the AFM tip to snap into contact with the sample surface. As a result, the most interesting range (a few nanometers above the sample surface) is left out [4] and the determination of the full tip-sample potential becomes impossible [3]. Therefore, the static measurement of the force-distance curve mainly serves to determine adhesion forces [4]. On the other hand, the AFM tip must get very close to the surface in order to achieve atomic resolution [2,9]. Dürig showed that the atomic-scale imaging can only be achieved when the tip-sample distance is less than $0.5 \mathrm{~nm}$ [9]. The "jump-to-contact" instability can be avoided by using a stiffer cantilever with the trade-off of a loss of sensitivity [3,5]. In comparison, tapping mode (TM) [10] can be used to avoid such instability with good sensitivity being reserved $[2,3,9]$. At the same time, TM can also dramatically reduce the sample damage during the scanning process [3,11]. Therefore, TM-AFM has been used to image and characterize various delicate biological samples such as antibody [12], protein [13], purple membrane [14,15] and virion [15]. Furthermore, the higher harmonics generated by the tip-sample

\footnotetext{
* Corresponding author. Tel.: +8610825 43970 .

E-mail address: zhangyin@lnm.imech.ac.cn (Y. Zhang).
} 
intermittent contact in TM-AFM can be used to enhance the signals $[11,14,16,17]$, which leads to the high resolution images and better mechanical characterization of the samples.

Understanding the tip-sample interaction is vital to the interpretation of the TM-AFM experimental data in terms of the topography and material property variations. The response displacement (together with time) of TM-AFM is recorded during the experiment and the tip-sample interaction is unknown. Finding the tip-sample interaction is thus an inverse problem [5,18-22]. In the theory aspect, there are two main sources making this inverse problem very difficult: (1) the AFM cantilever is a continuous system which has infinite degrees of freedom (DOF) and higher modes can participate in the motion; (2) the intermittent contact makes the system highly nonlinear, which manifests as the rich patterns of subharmonic motions and chaos [23-27]. Additionally, the noise contribution to the measurement and the large range of frequencies (bandwidth) needed to measure to recover the force also complicate this inverse problem [18]. It is impossible to solve this inverse problem without some assumptions [5]. By modeling the tip-sample interaction as an external force sensored by the AFM cantilever motion, this inverse problem can be greatly simplified and the nonlinear tip-sample interaction force can be directly given [28,29]. However, the tip-sample interaction as described by the Lennard-Jones (L-J) potential is displacement-dependent. The amplitude and frequency of an external force are by definition displacementindependent. Therefore, modeling the tip-sample interaction as an external force is inappropriate, especially when the tip is in contact with the sample [29]. Treating the AFM cantilever as one DOF point-mass-spring oscillator also greatly simplifies the problem $[5,14,22,28,29]$. The repulsive tip-sample interaction has much shorter acting range and time to AFM than those of the attractive ones such as vdW and Casimir forces. The repulsive tip-sample interaction acts as an impulse to excite the higher modes [30]. The excitation of higher modes upon impact is a characteristics of the continuous system, which is demonstrated in an impacting cantilever experiment [24]. The higher modes excited by the tip-sample interaction are also visible in the corresponding Fourier spectra [31]. When the tip-sample interaction is strong, multimodal analysis is needed to capture the AFM dynamics [32]. Of course, the excitation of higher modes can be inhibited or dramatically reduced in non-contact TM-AFM. However, short-ranged repulsive forces play a decisive role in high-resolution imaging: there pulsive forces acting on the tip apex are the major contribution to the gradient change (i.e., the contrast) while the attractive forces acting on the rest of tip form a background [3,9,33]. When TM-AFM is used to image biological samples in liquids, the response of AFM is intrinsically multimodal: the energy propagation between the modes even has more important contribution to the phase contrast image than the dissipated energy [15]. The multimodal analysis is a must for bimodal imaging [12,13,15], in which two driving frequencies around the two lowest eigenfrequencies of the AFM cantilever are deliberately applied to excite the two corresponding modes. The amplitude and phase shift of the first mode is to track the surface topography and those of the second mode are to detect the surface mechanical interaction [12,13]. In some previous multimodal analysis, modes are either de-coupled [29] or coupled only by the contact force [15]. Attard et al. [34] noticed that the AFM tip mass can significantly contribute to the whole system inertia. The influence of the concentrated mass on the system frequency can be very significant depending on the ratio of the concentrated mass to the whole system mass and its location [35], which has been utilized by Li et al. [11] to enhance the higher harmonics performance. The concentrated mass, contact damping and force as described by the Dirac delta function $[11,27,35]$ can all contribute to the mode coupling as shown in the multimodal analysis of this study.

The impact oscillator model [23-25,36] has been applied to study the intermittent contact dynamics of TM-AFM. In modeling aspect, the impact oscillator model requires the sample stiffness to be infinite to have an instantaneous impact [27]. The coefficient of restitution, which also contains the information of energy loss, relates the reflection velocity to the velocity just before the impact [23-25,36]. Obviously, the validity of the impact oscillator model depends on whether the impact and energy loss are instantaneous [25]. The tip-sample contact time of the TM-AFM in general is a considerable fraction of the cycle time [30], especially for a soft sample [27]. The impact oscillator model cannot be applied to the "tip-stuck-to-sample" motion [36], either. The grazing impact [37] study on TM-AFM is shown to have the characteristic features of an impact oscillator [25]. One outstanding feature in the grazing impact is that rather than integrating differential equations, the Poincaré mapping of algebraic equations can be derived to characterize the TM-AFM dynamics [24,25], which greatly simplifies the problem. However, the grazing impact is the zero velocity impact [37]. The viscous damping of the liquid bridge formed between the tip and sample may offer a mechanism for the zero velocity impact [25]. In general, the grazing impact cannot be used to study the AFM intermittent contact dynamics because of the nonzero impact velocity [27]. Hu and Raman [26] showed that the grazing impact model cannot capture their experimental results. In this study, a more general criterion of using the AFM tip displacement termed as switching condition [4,9,38,39] is adopted to tell whether the contact occurs. The Hertz and Derjaguin-Muller-Toporov (DMT) contact models here are used to model the tip-sample (repulsive) interaction instead of the Kelvin-Voigt model of a linearized spring [27,40]. The nonlinear force-displacement relation of the Hertz and DMT models leads to an asymmetric phase portrait of the "tipstuck-to-sample" motion as shown in this study, which cannot be captured by the Kelvin-Voigt model. All the models mentioned above only include the normal forces of the tip-sample interaction. The lateral friction can have significant even major contribution to the image [40]. The presence of friction may also lead to the wrong interpretation on the experimental data of the tip-sample interaction [41]. Mazeran and Loubet [42] showed the model deficiency of not considering the lateral friction by a comparison with the experimental data; they also concluded that the contrast of their force modulation microscope is mainly due to friction. Friction is also an important source of energy dissipation in TMAFM [43]. The AFM tip cannot be exactly perpendicular to the sample [44,45]. Furthermore, the AFM cantilever is often mounted with a tilting angle of $10-15^{\circ}$ relative to sample $[17,41]$ and the tilting angle dramatically increases the friction 
effect [41,42]. Kalinin et al. [46,47] modeled the friction as if the tip hits a linear spring. This study shows that the lateral friction acts as a pulsing axial force and bending moment on the AFM cantilever and its influence increases with the tilting angle. By assuming that the TM-AFM of intermittent contact is a weak nonlinear system and the AFM steady state is harmonic, Wang used Krylov-Bogolubov-Mitropolsky asymptotic method to show that the AFM steady-state response is a bistable one [48]. However, the intermittent contact can be highly nonlinear, which generates subharmonic motions and chaos [23-27]. Usually stronger tip-sample interaction makes larger distortion of harmonic and eventually destroys the harmonic motion. By shrinking the gap distance and keeping the driving force amplitude and frequency unchanged, which increases the tip-sample interaction, Stark [32] showed how the TM-AFM steady state evolves from a distorted harmonic motion, to subharmonic motion and finally to a nonperiodic motion. By using the displacement as the switching condition, there is no need for us to assume things such as instantaneous impact, zero velocity impact or weak nonlinearity and more general study on the TM-AFM intermittent contact dynamics can be done. Two types of TM-AFM motions: the intermittent contact and "tip-stuck-to-sample" motions are studied. Besides the inaccuracy of the harmonic motion assumption, we show that there are multiple steady states in intermittent contact due to different initial conditions, which makes the inverse problem solving much more difficult.

\section{Model development}

Fig. 1(a) shows the coordinate system and the AFM cantilever dimensions. The cantilever is with a length of $L$ and a tilting angle of $\theta$. In our coordinate system, the AFM cantilever and its tip at $x=x_{0}$ are separated from the sample with the distances of $d$ and $\delta / \cos \theta$, respectively. $g_{0}$ is the tip length. AFM is driven with a forced motion of $y_{0}(t)(t$ is time) at its fixed end. $w(x, t)$ is the beam deflection measured from the fixed end. The beam displacement $v(x, t)$ has the following expression:

$$
v(x, t)=w(x, t)+y_{0}(t)+d
$$

(a)
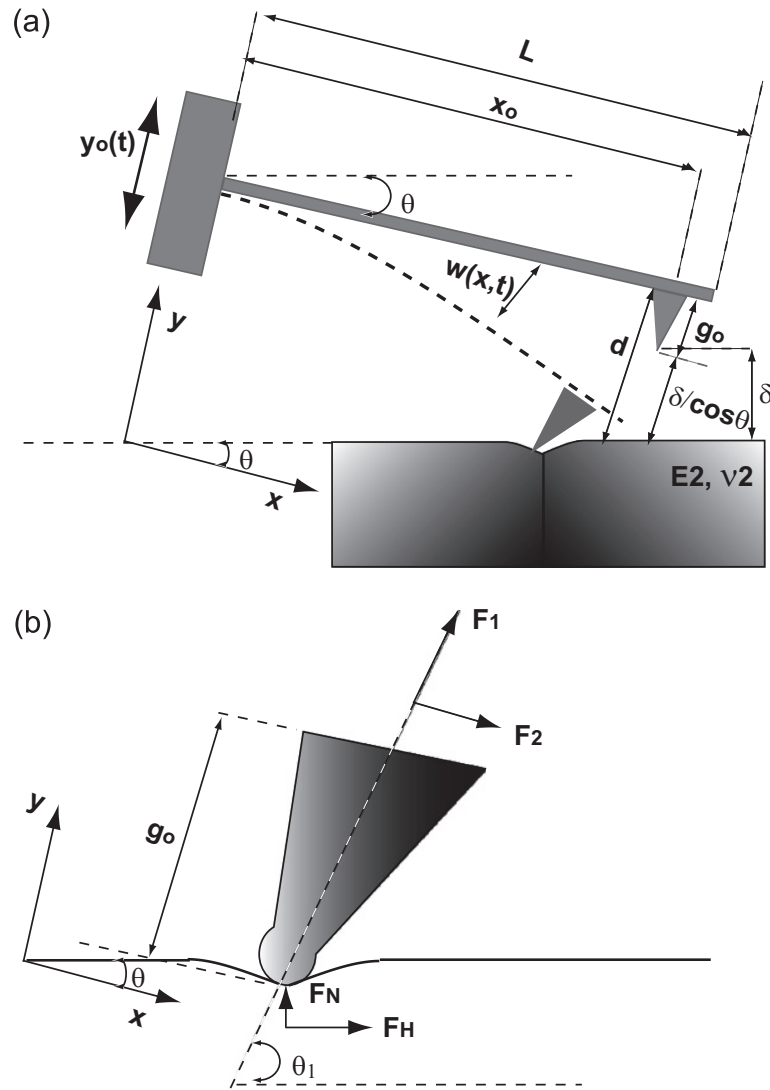

Fig. 1. Schematic diagrams of the AFM cantilever and sample. (a) The coordinate system and the beam dimensions. The cantilever length is $L$ and the tilting angle is $\theta$. The tip with a length of $d$ is located at $x=x_{0} ; \delta$ is the tip-sample separation distance. $y_{0}(t)$ is the oscillation at the cantilever fixed end; $w(x, t)$ is the beam deflection from the fixed end. (b) The tip end is modeled as a sphere with the radius of $R$ and the contact angle is $\theta_{1} ; F_{N}$ and $F_{H}$ are the normal and tangential forces due to the tip-sample contact, respectively; $F_{1}$ and $F_{2}$ are the transverse and axial component forces acting on the cantilever. 
Because $d=g_{0}+\delta / \cos \theta$, the beam tip displacement $v_{T}(t)=v\left(x_{0}, t\right)-g_{0}$ is expressed as follows:

$$
v_{T}(t)=w\left(x_{0}, t\right)+y_{0}(t)+\frac{\delta}{\cos \theta}
$$

As seen in Fig. 1(b) the tip end is modeled as a sphere with the radius of $R$. The contact angle, $\theta_{1}$, is readily derived as the following:

$$
\theta_{1}=\frac{\pi}{2}-\theta-\arctan \left(\left|\frac{\partial w}{\partial x}\right|_{x=x_{0}}\right) .
$$

For both the Hertz and Derjaguin-Muller-Toporov (DMT) models, the contact radius of $a$ is related with the normal indentation displacement of $v_{T} \sin \theta_{1}$ as follows [45,49]:

$$
\frac{a^{2}}{R}=-v_{T} \sin \theta_{1}
$$

$F_{N}$ is the normal contact force acting on the AFM tip, which is given as follows in conjunction with Eq. (4) [50,51]

$$
F_{N}= \begin{cases}\frac{4 E^{*} a^{3}}{3 R}=\frac{4 E^{*}\left(-v_{T} \sin \theta_{1} R\right)^{3 / 2}}{3 R} & \text { (Hertz), } \\ \frac{4 E^{*} a^{3}}{3 R}-2 \pi \gamma R=\frac{4 E^{*}\left(-v_{T} \sin \theta_{1} R\right)^{3 / 2}}{3 R}-2 \pi \gamma R & \text { (DMT), }\end{cases}
$$

where $E^{*}$ is the reduced Young's modulus and $1 / E^{*}=\left(1-v_{1}^{2}\right) / E_{1}+\left(1-v_{2}^{2}\right) / E_{2}\left(E_{1}, v_{1}\right.$ and $E_{2}, v_{2}$ are Young's moduli and Poisson's ratios of the tip and sample, respectively). $\gamma$ is the adhesion energy. $F_{H}$ is the tangential friction force acting on the AFM tip and is given as follows [52]:

$$
F_{H}=-8 G^{*} a\left(v_{T} \cos \theta_{1}\right)=-8 G^{*} \sqrt{-v_{T} \sin \theta_{1} R}\left(v_{T} \cos \theta_{1}\right),
$$

where $v_{T} \cos \theta_{1}$ is the tangential indentation displacement. $G^{*}$ is the reduced shear modulus and $1 / G^{*}=\left(2-v_{1}\right) / G_{1}+$ $\left(2-v_{2}\right) / G_{2} ; G_{1}=E_{1} / 2\left(1+v_{1}\right)$ and $G_{2}=E_{2} / 2\left(1+v_{2}\right)$ are the shear moduli of the tip and sample, respectively.

$F_{1}$ and $F_{2}$ are the two component forces acting transversely and axially on the AFM cantilever, which are readily given as follows:

$$
\left\{\begin{array}{l}
F_{1}=F_{N} \sin \theta_{1}+F_{H} \cos \theta_{1} \\
F_{2}=-F_{N} \cos \theta_{1}+F_{H} \sin \theta_{1}
\end{array}\right.
$$

For brevity, the governing equations derived by applying the Hamilton principle are given as follows:

$$
\begin{cases}{\left[M \delta_{D}\left(x-x_{0}\right)+m\right] \frac{\partial^{2} v}{\partial t^{2}}+C \frac{\partial v}{\partial t}+E_{1} I \frac{\partial^{4} v}{\partial x^{4}}=0,} & v_{T}>0, \\ {\left[M \delta_{D}\left(x-x_{0}\right)+m\right] \frac{\partial^{2} v}{\partial t^{2}}+C \frac{\partial v}{\partial t}+E_{1} I \frac{\partial^{4} v}{\partial x^{4}}-F_{2} \delta_{D}\left(x-x_{0}\right) \frac{\partial^{2} v}{\partial x^{2}}} & \\ -M_{b} \frac{\partial^{2}}{\partial x^{2}}\left[\delta_{D}\left(x-x_{0}\right)\right]-F_{1} \delta_{D}\left(x-x_{0}\right)+C_{1} \delta_{D}\left(x-x_{0}\right) \frac{\partial v}{\partial t}=0, & v_{T} \leq 0,\end{cases}
$$

where $M$ is the tip mass and $m$ is the mass per unit length of the beam. $I$ is the area moment of inertia and $I=b h^{3} / 12$ ( $b$ : beam width, $h$ : beam thickness) for a rectangular beam. $C$ is the viscous damping of the beam. $M_{b}$ is the bending moment due to the contact and $M_{b}=F_{2} d=\left(-F_{N} \cos \theta_{1}+F_{H} \sin \theta_{1}\right) d$. $C_{1}$ is the viscous damping due to the tip-sample contact, which is a damper in Kelvin-Voigt model [27,40,42]. $\delta_{D}$ is the Dirac delta function, which indicates that the tip mass is modeled as a concentrated mass [11,27] and tip-sample interaction is modeled as concentrated force and moment. $v_{T}$ defines the switching condition $[4,9,38,39] . v_{T}$ is a function of time $t . v_{T}=0$ is the criteria to tell whether the AFM tip is in contact with the sample or not for both the Hertz and DMT models. However, keep in mind that the contact/impact time is unknown. Even when the analytical solutions before and after impact are available to a piecewise linear impact oscillator, the unknown property of impact time still makes the system nonlinear [39,44]. It is also worth mentioning that the tip-sample interaction forces derived by the two contact models are the short-range repulsive forces, which play a decisive role in the AFM imaging $[3,9,33]$. Furthermore, because the long-rang attractive vdW force does not contribute to the energy loss of the TM-AFM, its influence on the AFM dynamics is ignored [25,27].

$y(t)$ is a sinusoidal oscillation given as follows:

$$
y(t)=f \sin (\omega t),
$$

where $f$ and $\omega$ are the driving amplitude and frequency, respectively. The following dimensionless quantities are introduced to nondimensionalize equation (8)

$$
\xi=\frac{x}{L}, \quad \tau=\sqrt{\frac{E_{1} I}{m L^{4}}} t, \quad W=\frac{w}{d}, \quad V=\frac{v}{d}, \quad V_{T}=\frac{v_{T}}{d}, \quad \Omega=\sqrt{\frac{m L^{4}}{E_{1} I}} \omega .
$$


$\sqrt{E_{1} I / m L^{4}}$ is with the unit of Hertz and it is the same order of the first natural frequency of a uniform and undamped cantilever beam [27,53]. In conjunction with Eqs. (5)-(7) and (9), Eq. (8) now becomes the following dimensionless ones:

$$
\begin{cases}{\left[\alpha_{1} \delta_{D}\left(\xi-\xi_{0}\right)+1\right] \frac{\partial^{2} W}{\partial \tau^{2}}+\alpha_{3} \frac{\partial W}{\partial \tau}+\frac{\partial^{4} W}{\partial \xi^{4}}=\left[\alpha_{1} \delta_{D}\left(\xi-\xi_{0}\right)+1\right] \alpha_{2} \Omega^{2} \sin (\Omega \tau)-\alpha_{2} \alpha_{3} \Omega \cos (\Omega \tau),} & V_{T}>0, \\ {\left[\alpha_{1} \delta_{D}\left(\xi-\xi_{0}\right)+1\right] \frac{\partial^{2} W}{\partial \tau^{2}}+\alpha_{3} \frac{\partial W}{\partial \tau}+\alpha_{4} \delta_{D}\left(\xi-\xi_{0}\right) \frac{\partial W}{\partial \tau}+\frac{\partial^{4} W}{\partial \xi^{4}}-\left[-\alpha_{5} f_{c} \cos \theta_{1}+\alpha_{6} A\left(-V_{T} \cos \theta_{1}\right) \sin \theta_{1}\right] \frac{\partial^{2} W}{\partial \xi^{2}}} & \\ = & {\left[\alpha_{1} \delta_{D}\left(\xi-\xi_{0}\right)+1\right] \alpha_{2} \Omega^{2} \sin (\Omega \tau)-\alpha_{2} \alpha_{3} \Omega \cos (\Omega \tau)-\alpha_{2} \alpha_{4} \delta_{D}\left(\xi-\xi_{0}\right) \Omega \cos (\Omega \tau)} \\ & +\frac{L}{d}\left[\alpha_{5} f_{c} \sin \theta_{1}+\alpha_{6} A\left(-V_{T} \cos \theta_{1}\right) \cos \theta_{1}\right] \delta_{D}\left(\xi-\xi_{0}\right)+\frac{g_{0}}{d}\left[-\alpha_{5} f_{c} \cos \theta_{1}+\alpha_{6} A\left(-V_{T} \cos \theta_{1}\right) \sin \theta_{1}\right] \frac{\partial^{2} \delta_{D}\left(\xi-\xi_{0}\right)}{\partial \xi^{2}}, \quad V_{T} \leq 0,\end{cases}
$$

where $A=\sqrt{-V_{T} \sin \theta_{1} / \beta}$ is the dimensionless contact radius; $\beta$ is a dimensionless parameter defined as $\beta=$ $\left((9 \pi / 8) \gamma / E^{*} d\right)^{2 / 3} \times(R / d)^{1 / 3} ; f_{c}=A^{3}$ for the Hertz model and $f_{c}=A^{3}-4 / 3$ for the DMT model. Here dimensionless $\alpha_{i}$ 's $(i=1$ to 6$)$ are defined as

$$
\alpha_{1}=\frac{M}{m L}, \quad \alpha_{2}=\frac{f}{d}, \quad \alpha_{3}=C \sqrt{\frac{L^{4}}{E_{1} I m}}, \quad \alpha_{4}=\frac{C_{1}}{L} \sqrt{\frac{L^{4}}{E_{1} m}}, \quad \alpha_{5}=\frac{3 \pi \gamma R L^{2}}{E_{1} I}, \quad \alpha_{6}=\frac{8 G^{*} d R L^{2}}{E_{1} I}\left(\frac{9 \pi \gamma}{E^{*} R}\right)^{1 / 3} .
$$

Physically $\alpha_{1}$ indicates the ratio of the tip mass to the whole beam mass and $\alpha_{2}$ indicates the ratio of the driving amplitude to the cantilever separation distance. $\alpha_{4}$ and $\alpha_{3}$ indicate the damping influence with and without the contact with the sample, respectively. $\pi \gamma R$ and $R\left(9 \pi \gamma / E^{*} R\right)^{1 / 3}$ are the same order of the pull-off force and contact radius [51,52], therefore, $\alpha_{5}$ and $\alpha_{6}$ indicate the influence of normal and shear force due to adhesion compared with the elastic one $\left(E_{1} I / L^{2}\right)$. In Eq. (11), $\left[\alpha_{1} \delta_{D}\left(\xi-\xi_{0}\right)+1\right] \alpha_{2} \Omega^{2} \sin (\Omega \tau)-\alpha_{2} \alpha_{3} \Omega \cos (\Omega \tau)$ is the de facto driving force. Physically the dither piezo supplies a sinusoidal motion at the cantilever end as described by Eq. (9) [4]. However, many theories model that the dither piezo applies a sinusoidal driving force of $\alpha_{2} \Omega^{2} \sin (\Omega \tau)$ to the system and the deficiency of such modeling, especially when $\alpha_{3}$ is large, has already been pointed out by Hölscher and Schwarz [4].

The switching condition of Eq. (2) is now nondimensionalized as follows:

$$
V_{T}(\tau)=W\left(\xi_{0}, \tau\right)+\alpha_{2} \sin (\Omega \tau)+\frac{\delta}{d \cos \theta} .
$$

Here the Galerkin method is used for the computation of Eq. (11) and $W(\xi, \tau)$ is discretized as

$$
W(\xi, \tau)=\sum_{j=1}^{N} a_{j}(\tau) \phi_{j}(\xi)
$$

$a_{j}(\tau)$ is the unknown modal amplitude to be determined and $N$ is the mode number. $\phi_{j}(\xi)$ is the modal shape, or say, mode of a uniform cantilever beam [53], which mathematically is the eigenvector associated with the jth eigenfrequency. Substitute Eq. (14) into Eq. (11), time $\phi_{i}(\xi)$ and integrate from 0 to 1, the following governing equations are derived:

$$
\begin{cases}\mathbf{M}^{\mathrm{I}} \ddot{\mathbf{X}}+\mathbf{C}^{\mathrm{I}} \dot{\mathbf{X}}+\mathbf{K}^{\mathbf{I}} \mathbf{X}=\mathbf{F}^{\mathbf{I}}, & V_{T}>0 \\ \mathbf{M}^{\mathrm{II}} \ddot{\mathbf{X}}+\mathbf{C}^{\mathrm{II}} \dot{\mathbf{X}}+\mathbf{K}^{\mathrm{II}} \mathbf{X}=\mathbf{F}^{\mathrm{II}}, & V_{T} \leq 0 .\end{cases}
$$

Here $(\dot{)})=\partial / \partial \tau, \mathbf{X}=\left(a_{1}, a_{2}, \ldots, a_{N}\right)^{T}, \mathbf{F}^{\mathbf{I}}=\left(\mathbf{F}_{\mathbf{1}}^{\mathbf{I}}, \mathbf{F}_{2}^{\mathbf{I}}, \ldots, \mathbf{F}_{\mathbf{N}}^{\mathbf{I}}\right)^{\mathbf{T}}$ and $\mathbf{F}^{\mathbf{I I}}=\left(\mathbf{F}_{\mathbf{1}}^{\mathbf{I I}}, \mathbf{F}_{\mathbf{2}}^{\mathbf{I I}}, \ldots, \mathbf{F}_{\mathbf{N}}^{\mathbf{I I}}\right)^{\mathbf{T}}$. During the integration, the orthogonality property of the mode shapes and integration property of the Dirac delta function are used [35]. Matrices $\mathbf{M}^{\mathbf{I}}, \mathbf{C}^{\mathbf{I}}$ and $\mathbf{K}^{\mathbf{I}}$ are derived as follows:

$$
\begin{gathered}
\mathbf{M}_{i j}^{\mathbf{I}}= \begin{cases}\alpha_{1} \phi_{i}(1) \phi_{i}(1)+\int_{0}^{1} \phi_{i}(\xi) \phi_{i}(\xi) \mathrm{d} \xi, & i=j, \\
\alpha_{1} \phi_{i}(1) \phi_{j}(1), & i \neq j,\end{cases} \\
\mathbf{C}_{i j}^{\mathbf{I}}= \begin{cases}\alpha_{3} \int_{0}^{1} \phi_{i}(\xi) \phi_{i}(\xi) \mathrm{d} \xi, & i=j, \\
0, & i \neq j,\end{cases}
\end{gathered}
$$

and

$$
\mathbf{K}_{i j}^{\mathbf{I}}= \begin{cases}\int_{0}^{1} \phi_{i}(\xi) \frac{\partial^{4} \phi_{i}(\xi)}{\partial \xi^{4}} \mathrm{~d} \xi, & i=j, \\ 0, & i \neq j .\end{cases}
$$

Due to the integration property of the Dirac delta function, $\mathbf{M}^{\mathbf{I}}$ is not diagonal, which couples the modes even for small linear vibration. Vector $\mathbf{F}^{\mathbf{I}}$ is given as the following:

$$
\mathbf{F}_{\mathbf{i}}^{\mathbf{I}}=\alpha_{2} \Omega^{2}\left[\alpha_{1} \phi_{i}(1)+\int_{0}^{1} \phi_{i}(\xi) \mathrm{d} \xi\right] \sin (\Omega \tau)-\alpha_{2} \alpha_{3} \Omega \int_{0}^{1} \phi_{i}(\xi) \mathrm{d} \xi \cos (\Omega \tau) .
$$


$\mathbf{M}^{\text {II }}, \mathbf{C}^{\text {II }}$ and $\mathbf{K}^{\mathrm{II}}$ are

$$
\begin{gathered}
\mathbf{M}^{\mathbf{I}}=\mathbf{M}^{\mathbf{I}}, \\
\mathbf{C}_{i j}^{\mathrm{II}}= \begin{cases}\alpha_{3} \int_{0}^{1} \phi_{i}(\xi) \phi_{i}(\xi) \mathrm{d} \xi+\alpha_{4} \phi_{i}\left(\xi_{0}\right) \phi_{i}\left(\xi_{0}\right), & i=j, \\
\alpha_{4} \phi_{i}\left(\xi_{0}\right) \phi_{j}\left(\xi_{0}\right), & i \neq j,\end{cases}
\end{gathered}
$$

and

$$
\mathbf{K}_{i j}^{I I}= \begin{cases}\int_{0}^{1} \phi_{i}(\xi) \frac{\partial^{4} \phi_{i}(\xi)}{\partial \xi^{4}} \mathrm{~d} \xi-\left[-\alpha_{5} f_{c} \cos \theta_{1}+\alpha_{6} A\left(-V_{T} \cos \theta_{1}\right) \sin \theta_{1}\right] \int_{0}^{1} \phi_{i}(\xi) \frac{\partial^{2} \phi_{i}(\xi)}{\partial \xi^{2}} \mathrm{~d} \xi, & i=j, \\ -\left[-\alpha_{5} f_{c} \cos \theta_{1}+\alpha_{6} A\left(-V_{T} \cos \theta_{1}\right) \sin \theta_{1}\right] \int_{0}^{1} \phi_{i}(\xi) \frac{\partial^{2} \phi_{i}(\xi)}{\partial \xi^{2}} \mathrm{~d} \xi, & i \neq j,\end{cases}
$$

Keep in mind that $\phi_{i}(\xi)$ and $\partial^{2} \phi_{i}(\xi) / \partial \xi^{2}$ are not orthogonal to each other. As seen in Eqs. (21) and (22), the contact damping couples the modes because of the Dirac delta function and the contact force couples the modes because of its axial component. $\mathbf{F}^{\text {II }}$ is given as follows:

$$
\mathbf{F}_{i}^{\mathbf{I I}}=\mathbf{F}_{i}^{\mathbf{I}}-\alpha_{2} \alpha_{4} \phi_{i}\left(\xi_{0}\right) \sin (\Omega \tau)+\frac{L}{d}\left[\alpha_{5} f_{c} \sin \theta_{1}+\alpha_{6} A\left(-V_{T} \cos \theta_{1}\right) \cos \theta_{1}\right] \phi_{i}\left(\xi_{0}\right)+\frac{g_{0}}{d}\left[-\alpha_{5} f_{c} \cos \theta_{1}+\alpha_{6} A\left(-V_{T} \cos \theta_{1}\right) \sin \theta_{1}\right] \frac{\partial^{2} \phi_{i}}{\partial \xi^{2}}\left(\xi_{0}\right)
$$

The tip-sample interaction further couples the modes as $\mathbf{F}_{i}^{\mathbf{I I}}$ is a function of $V_{T}$. A Fortran program of the fourth-order Runge-Kutta integration [54] is used for the time integration of Eq. (15). It is also worth pointing out that because $V_{T}(\tau)$ and $\theta_{1}$ are the functions of time, $\mathbf{K}^{\mathrm{II}}$ needs to be updated in each time step during the contact. The system can have significant difference in stiffness before and after contact, which changes the system eigenfrequencies [27,42,46,47]. To achieve the same accuracy for the system before and after contact, the time step is different. It is well possible that the integration routine with large time step can overshoot the discontinuity to cause the computational inaccuracy [27]. To avoid such scenario is either to construct the time grid which reduces dramatically as the AFM tip approaches the sample and keeps small during contact [27] or just to take very small time step for both contact and non-contact regions. In our computation the time step before contact is taken as $\mathrm{d} \tau=2 \times 10^{-3}$ and the time step during contact is taken as $\mathrm{d} \tau / 16$.

\section{Results and discussion}

The following dimensions for a typical AFM are taken as $L=300 \mu \mathrm{m}, h=3 \mu \mathrm{m}, b=30 \mu \mathrm{m}, R=20 \mathrm{~nm}$ [55,56]. The AFM is made of single crystal silicon with $E_{1}=130 \mathrm{GPa}$ and $v_{1}=0.27[55,56]$; the sample is polystyrene with $E_{2}=1.2 \mathrm{GPa}, v_{2}=0.3$ and $\gamma=3.5 \times 10^{-2} \mathrm{~J} / \mathrm{m}^{2}$ [56]. Notice that $\gamma$ here does not account for the surface energy hysteresis. The surface energy hysteresis physically indicates that the energy obtained by combining two surfaces into one interface is less than that required to separate one interface into two surfaces, which is also an energy dissipating mechanism [43]. We also set the following parameters as $\theta=\pi / 12[17,41]$ and $d=g_{0}=5 \mu \mathrm{m}$ (therefore $\delta=0 \mu \mathrm{m}$ ). The corresponding dimensionless parameters are thus computed as

$$
\alpha_{5}=6.7665 \times 10^{-5}, \quad \alpha_{6}=0.7413, \quad \beta=1.128 \times 10^{-4}, \quad \frac{L}{d}=60, \quad \frac{g_{0}}{d}=1
$$

$\alpha_{1}, \alpha_{3}, \alpha_{4}$ and $\Omega$ are set as $\alpha_{1}=0.05, \alpha_{3}=\alpha_{4}=0.4$ and $\Omega=2$. The vibration of AFM in air has high $Q$ factor, or say small damping [27]. As indicated in Eq. (12), the system is an underdamped system by setting $\alpha_{3}=\alpha_{4}=0.4$. The above parameters apply to all the figures presented here. The tip-sample interaction is modulated by changing $\alpha_{2}$, which is the ratio of the driving amplitude to the cantilever separation distance defined as f/d in Eq. (12). $\alpha_{2}=5 \times 10^{-4}$ for Figs. $2-6$ and $\alpha_{2}=1 \times 10^{-3}$ for Fig. 7. Eqs. (5) and (6) are derived from the elasticity theory which assumes that the contact radius is very small compared with $R$ [52]. The reason to take those small $\alpha_{2}$ s is to make sure that the indentation depth is very small (so is the contact radius), Eqs. (5) and (6) can thus hold. It is worth pointing out that Eqs. (5) and (6) are the contact statics, which may not be valid when $\eta_{2}=f \omega / \sqrt{G_{2} / \rho_{2}} \geq 0.1$ ( $\rho_{2}$ is the mass density of sample) and $\sqrt{G_{2} / \rho_{2}}$ is the shear wave velocity inside the sample) [57]. In our case $\eta_{2} \approx 10^{-8}$, it is thus reasonable to have this quasi-static model.

The first three dimensionless eigenfrequencies of a uniform cantilever are [27,53]

$$
\Omega_{01}=1.875^{2}=3.52, \quad \Omega_{02}=4.694^{2}=22.03, \quad \Omega_{03}=7.855^{2}=61.7 .
$$

The driving frequency is $\Omega=2$, which is below the first eigenfrequency and the first mode shape is supposed to dominate. However, the convergence study of Fig. 2 on the steady-state motion shows that it is not true. As the mode number $N$ increases from 1 to 4 , the significant difference between $N=1$ and the others is clearly seen. There is little difference between $N=2$ and $N=3$ and there is almost no difference between $N=3$ and $N=4$. $V_{T}$ is the dimensionless beam tip displacement and $V_{T} \leq 0$ is the contact state. As seen in Fig. 2, the contact time constitutes a significant portion of the driving period. Physically the motion difference computed by different mode number is caused by the participation of the higher modes into the motion. The one DOF model which obtains the AFM stiffness by statics in essence can only be 


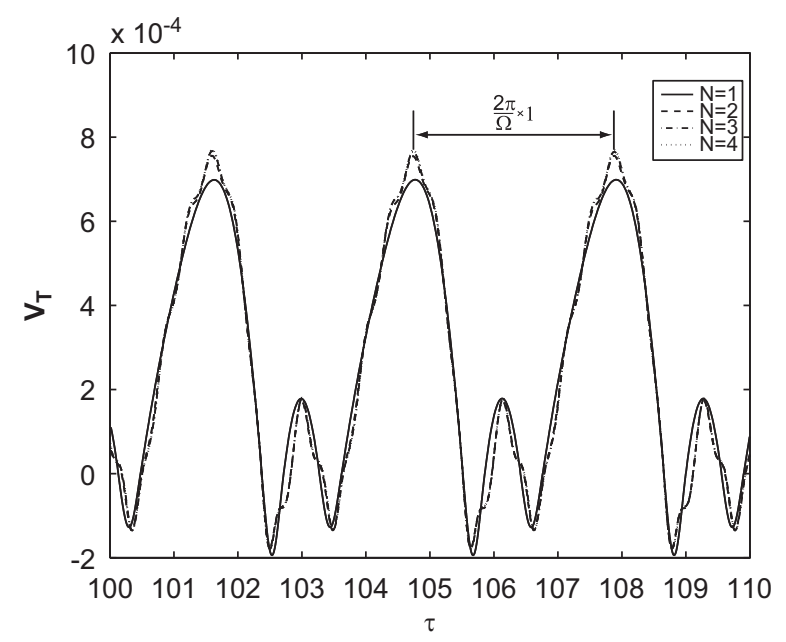

Fig. 2. The convergence study on the mode number $(N)$ when $\Omega=2$ and $\alpha_{2}=5 \times 10^{-4}$. $N$ is taken as $1,2,3$ and 4 , respectively. The results converge when $N \geq 3$.
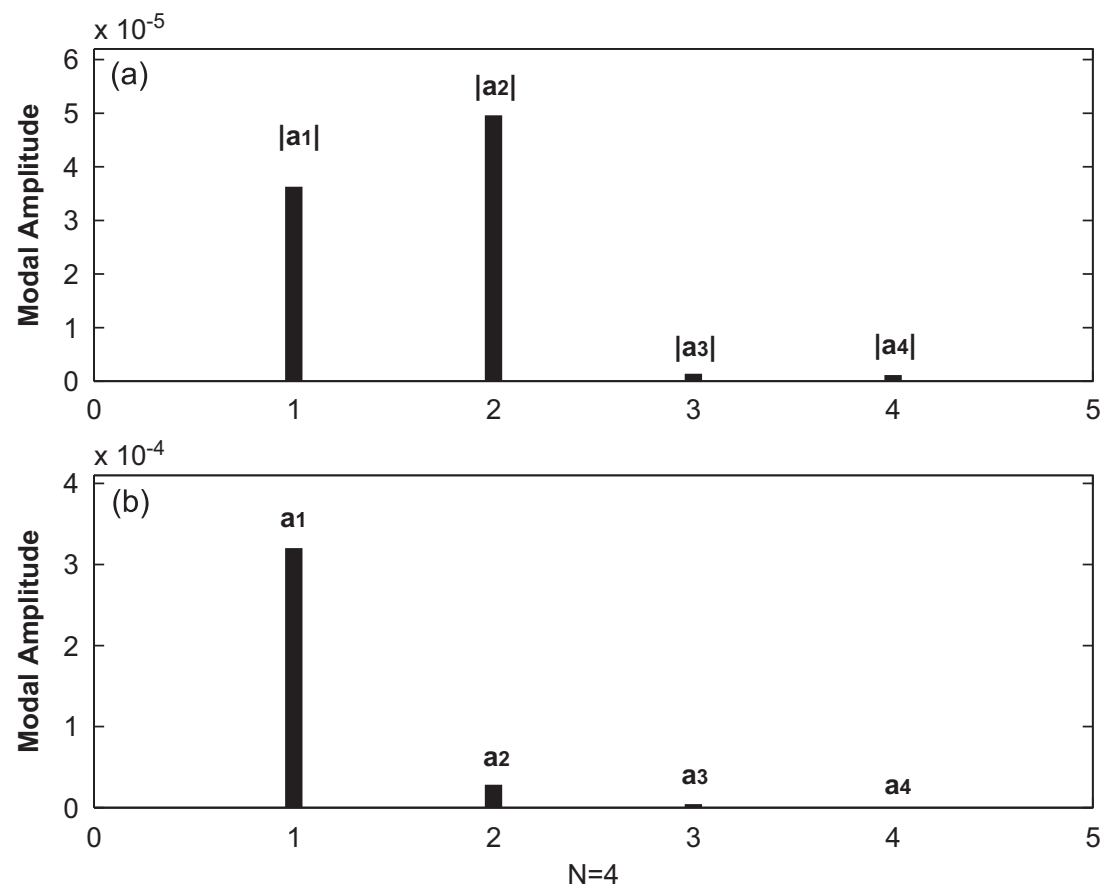

Fig. 3. (a) The modal amplitudes when the tip is in contact with the sample. The amplitude of the second mode is even larger than that of the first one. (b) The modal amplitudes when the tip is not in contact with the sample. The first mode dominates. There are total four modes $(N=4)$ used in the computation.

accurate when there is no higher modes participation in the motion. When the driving frequency is much higher than the first eigenfrequency [23], the equivalent stiffnesses for higher modes are obtained by a rather complex statics approach [58]. Here the multimodal analysis presents a simple and more importantly, a systematic way of generating the stiffness matrix. As seen in Fig. 2, because the intermittent contact induces the higher modes in the motion even when the driving frequency is well below the first eigenfrequency, the multimodal analysis and its convergence study are needed in the TM-AFM dynamics. Compared with the fast Fourier transform (FFT) analyses which only study the power spectrum of overall motion (including both contact and non-contact motions) [23,32], the multimodal analysis here allows us to differentiate the modal amplitudes before and after contact as presented in Fig. 3 with $N=4$. In the contact state the amplitude of the second mode is even larger than that of the first mode; the third and fourth modes, though small, are clearly excited, which is the reason why the results in Fig. 2 converge when $N \geq 3$. In the non-contact state, the first mode, as expected, dominates with some minor participation of the second mode. The participation of the second mode in the 

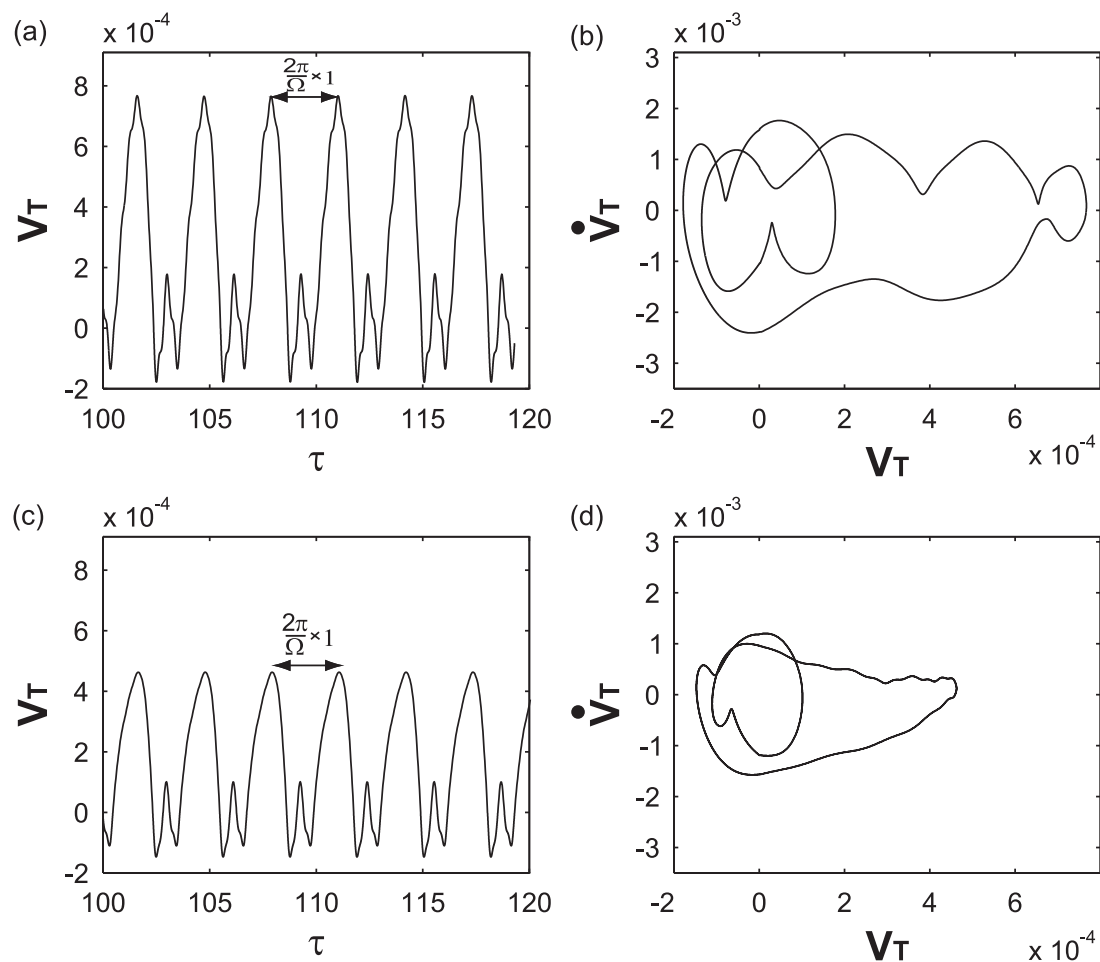

Fig. 4. The time series and phase portraits of two period-1 motions with $\alpha_{2}=5 \times 10^{-4}$; (a) and (c) are the time series; (a) is the motion presented in Fig. 2. (b) and (d) are the phase portraits.
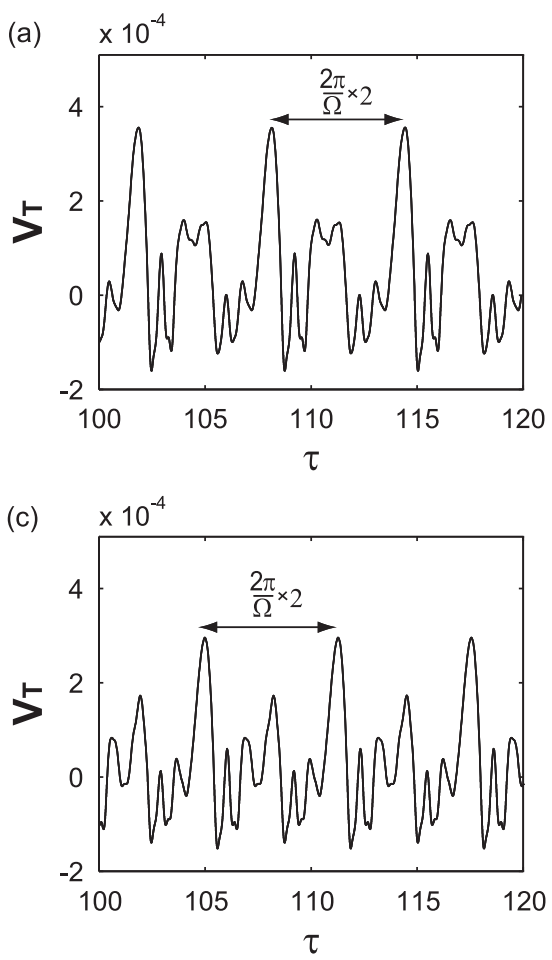
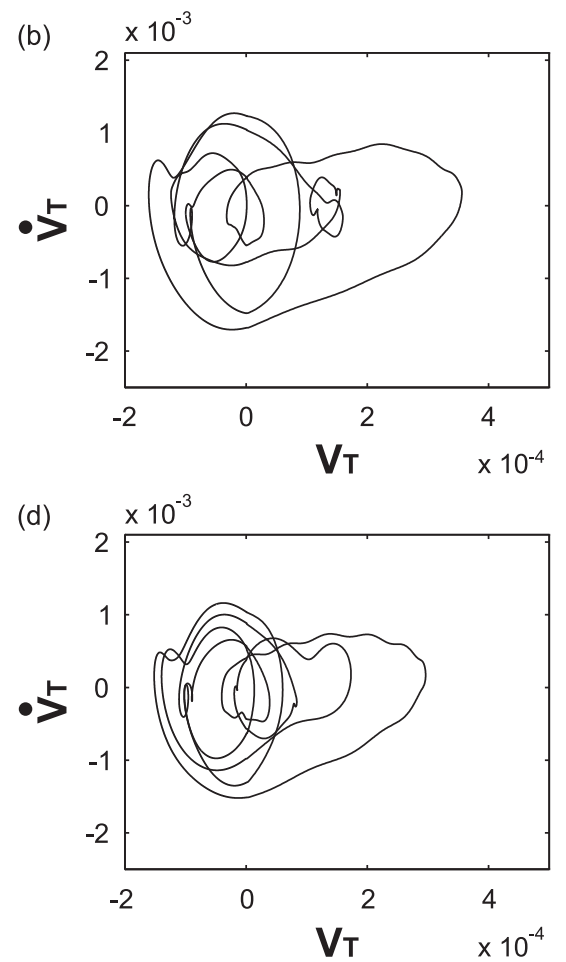

Fig. 5. The time series and phase portraits of two period-2 motions with $\alpha_{2}=5 \times 10^{-4}$. (a) and (c) are the time series; (b) and (d) are the phase portraits. 


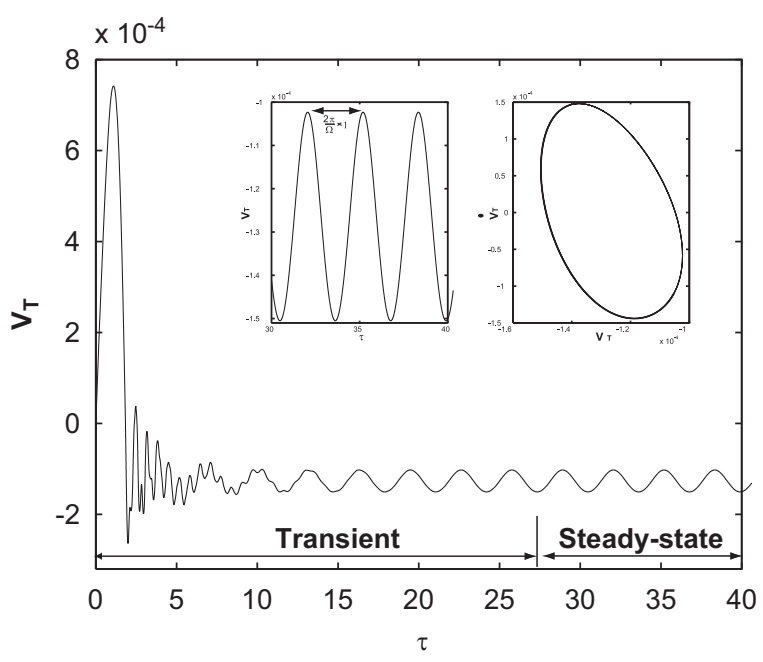

Fig. 6. The time series of the adhesive DMT contact, which share the same initial conditions as those of the non-adhesive Hertz contact presented in Fig. 2 and $\alpha_{2}=5 \times 10^{-4}$. The inset is a closer look at its time series and phase portrait of the steady state. In the steady state, the tip is always in contact with the sample, i.e., no intermittent contact occurs.

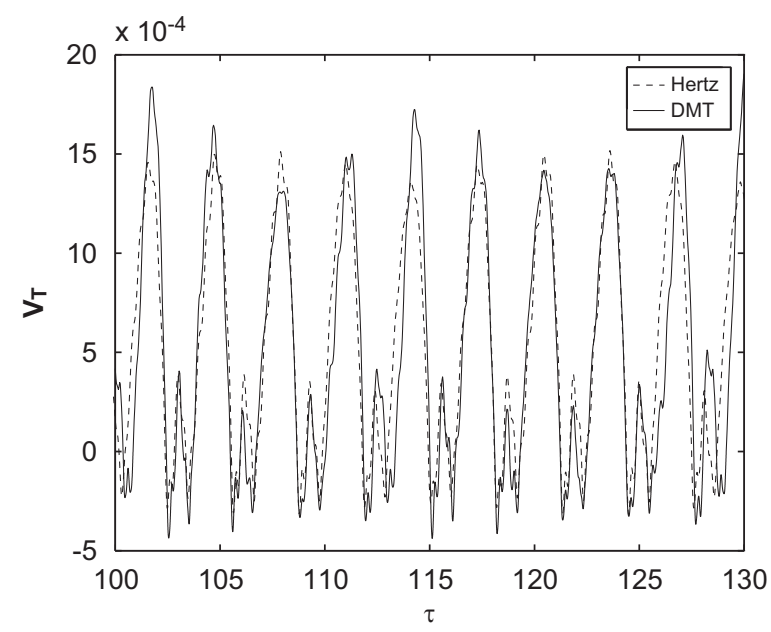

Fig. 7. Time series comparison of the Hertz and DMT models with $\alpha_{2}=1 \times 10^{-3}$. Both motions are non-periodic.

non-contact state is due to the two reasons: (1) the tip mass gives the non-diagonal element in the mass matrix of Eq. (16) and thus couple the modes; (2) the higher modes excited in the contact state does not die out in a forcing period because of the underdamped property of the system. As for the contact state, the tip mass, the tip-sample damping and the contact forces are all the sources for coupling the modes as seen in Eqs. (20)-(23). Because of the elastic restoring force due to contact, the system stiffens significantly, which is reflected by the fact that $a_{1}$ of non-contact state is almost 10 times larger than $a_{1}$ and $a_{2}$ of contact state.

Subharmonic is the period- $n$ oscillation that takes $n$ forcing periods to complete a full cycle [27]. Figs. 4 and 5 present the time series and phase portraits of two different period- 1 and period- 2 steady-state motions, respectively. The motions are computed with $N=3$ and $\dot{V}_{T}$ is the dimensionless velocity of beam tip. As for initial conditions, all the initial modal amplitude and velocity are set zero except $a_{1}$. In Figs. 4(a), (c), 5(a) and (c), the initial $a_{1}$ is set as $0,3 \times 10^{-4}, 7 \times 10^{-4}$ and $8 \times 10^{-4}$, respectively. There are other subharmonic motions which are due to different initial conditions and not presented here. The period-2 motions presented in Fig. 5 are also called period-doubling [28,30]. Figs. 4 and 5 clearly demonstrate that due to different initial conditions, different motion patterns can appear under the same governing equations. As an AFM scans from one area to another, the initial conditions vary, which may generate different steadystate motions. There are two main sources contributing to the system nonlinearity: (1) The contact forces of Eqs. (5) and (6) are the nonlinear functions of $V_{T}$; furthermore, the axial force due to contact acts as a pulsing force which constantly changes the system stiffness during the contact. (2) The intermittent contact itself $[39,44]$. The driving 
frequency here is quite low. With the increase of driving frequency, both experiment [23] and simulation [27] show that the period- $n$ subharmonic motion with larger $n$ appears.

The non-adhesive Hertz contact model is used in Figs. 2-5. Now let us examine the adhesion influence and here the DMT model is used. All the parameters and initial conditions in Fig. 6 are the same as those in Fig. 2. As seen in Fig. 6, after one bouncing off the sample, the AFM tip is in contact with sample all the time, i.e., $V_{T}$ is always a negative value and thus no intermittent contact occurs. Compared with Fig. 2, the motion of adhesive contact in Fig. 6 is a quite different one. This is the case of "tip-stuck-to-sample" by adhesion [36], in which the "coefficient-of-restitution" rule [36] and the mapping study [25] of the impact oscillator model become invalid. Compared with the Hertz model with $V_{T}=0$ as the equilibrium, the DMT equilibrium is a negative one because of adhesion. As seen in Eq. (6), the force due to adhesion, $-2 \pi \gamma R$, acts as an external attractive force to drag the AFM tip into contact [50,51]. $-2 \pi \gamma R$ is also called pull-off force, which is the external tensile force required to separate the sphere tip from contact [51]. $\alpha_{2}=5 \times 10^{-4}$ is a small vibration amplitude, which is not large enough to separate the tip from the sample to make the intermittent contact. The insets provide a closer look at its steady-state motion, which is a period-1 motion and its phase portrait is an asymmetric one. The reason for the asymmetric phase portrait is that the restoring elastic contact forces are nonlinear functions of $V_{T}$ as if the tip hits a nonlinear spring. This asymmetry cannot be captured if a linearized spring model is applied to model the contact [27,40]. Besides the pulsing axial force, the nonlinear relation of the tip-sample relation can introduce displacement-dependent eigenfrequency, which is also found by Jesse et al. [46] that the tip position can significantly affect the resonance spectrum. Before we have any further discussion, it is worthwhile for us to differentiate the higher harmonics and higher modes. The higher harmonics referred in this paper are the integer multiples of the forcing frequency $\Omega$. Except for a hinged-hinged beam, the eigenfrequencies of a beam are not consecutive integer multiple, or harmonics, of each other. As a result, the cantilever beam modes associated with the eigenfrequencies are not harmonics. For example, in Eq. $(25)$, $\Omega_{02} / \Omega_{01} \approx 6.26$ and $\Omega_{03} / \Omega_{01} \approx 17.53$ for a uniform cantilever beam. By manipulating the tip mass and its location, Li et al. [11] made $\Omega_{02} / \Omega_{01}=5$ and $\Omega_{03} / \Omega_{01}=15$; or by cutting a hole in the beam, Sahin et al. [16] made $\Omega_{03} / \Omega_{01}=16$. Therefore, the signals of those higher harmonics which coincide with the higher eigenfrequencies can be significantly enhanced due to resonance when driving frequency is around the first eigenfrequency [11,16]. Balantekin and Atalar [17] used a more direct approach to make the first eigenfrequency the higher harmonics by driving the AFM cantilever around $1 / 3$ and $1 / 2$ of the first eigenfrequency. Keep in mind the above enhancing mechanisms of making the eigenfrequency a higher harmonics of the driving frequency implicitly assume the tip-sample interaction as an external force, which does not change the system eigenfrequencies. The tip-sample interaction is displacement-dependent, or say, surface-coupled [47], which can significantly change the system eigenfrequencies [27,42,46,47]. As seen in Fig. 2 the one mode computation has qualitatively captured the motions. The two peaks in one driving period of $2 \pi / \Omega$ is mainly due to the participation of higher harmonics not the higher modes. The higher harmonics result from the nonlinear tip-sample interactions and as pointed out by Crittenden et al. [59], the nonlinearity is manifested not in the mode shape but rather in the temporal dynamics of the mode shape. Our system is an underdamped one, the small damping, or say, high quality factor, prevents the energy transfer between modes [15], which allows us to catch the basic temporal dynamics in one mode. When TM-AFM is placed in an overdamped environment of liquid, one mode analysis breaks down [15]. The higher harmonics participation further stands out in the period-2 motions as presented in Fig. 5, in which there are several peaks in one driving period. Without intermittent contact, Fig. 6 has only one peak in one driving period, which means that much less higher harmonics participate in the motion. Unlike that in Fig. 2, the contribution of the second mode and other higher modes in Fig. 6 is negligible in both the contact and non-contact states. Also unlike the intermittent contact which is sensitive to the initial conditions as presented in Figs. 4 and 5, the steady state of the "tip-stuck-to-sample" motion seems unique with different initial conditions. The dominance of the first mode, much less higher harmonics participation and in sensitiveness to the initial conditions of the "tip-stuck-to sample" motion should be of a great help to solving the inverse problem.

Now $\alpha_{2}$ is increased to $\alpha_{2}=1 \times 10^{-3}$ to have intermittent contact and stronger tip-sample interaction. Again, $\alpha_{2}$ is the ratio of the driving amplitude to the cantilever separation distance defined as $f / d$ in Eq. (12). The two motions described by the Hertz and DMT models share the same parameters and initial conditions. Both motions are nonperiodic as plotted in Fig. 7. Although chaos is frequently encountered in the AFM intermittent contact dynamics [24-26], we should be cautious not to conclude that those nonperiodic motions are chaos. Because several period- $n$ motions can coexist at one driving frequency [27], the nonperiodic motion can be the transitional results that the motion of period- $m$ evolves to the motion of period- $n$, or the motion itself oscillates between the orbits even the time lasts long enough $(\tau \geq 100)$. Actually the motion of the Hertz model seems on the way of evolving to a period-4 motion. One pattern observed in these two nonperiodic motions is that the DMT motion always has larger indentation, i.e., $V_{T}^{D M T} \leq V_{T}^{H E R T Z} \leq 0$. Again, this is due to the adhesion force which always makes the contact radius (so is the indentation) of adhesive contact larger than that of non-adhesive Hertz contact. Again, because of the intermittent contact, there are two peaks in one driving period, which indicates the participation of higher harmonics. As mentioned above there are two major sources causing the nonlinearity and it seems that the intermittent contact plays a much more important role than the nonlinearity of the contact force-displacement relation. A vital assumption used in solving the inverse problem in Refs. [19-22] is that the cantilever steady state is harmonic, i.e., $v_{T}(t)=\delta+B+B \sin (\omega t+\psi)$ [21], which is more specifically called by Stark et al. [28] as the assumption of a disturbed harmonic oscillation. Here $B$ and $\psi$ are the mean amplitude and phase shift, in which the information on the tipsample interaction is contained. The assumption of harmonic motion can only be valid when the tip-sample interaction 
is(very) weak [19]. However, the participation of higher harmonics and modes often makes the motion anharmonic [29,31] because the repulsive tip-sample interaction (i.e., the force due to the tip-sample elastic deformation) is usually strong, which leads to that the theory based on the assumption of harmonic motion deviates significantly from the experimental observation [20]. As also seen in Figs. 4, 5 and 7, the AFM steady-state motion cannot be approximated by a harmonic motion.

\section{Conclusions}

A comprehensive dynamics model on a tilted AFM is presented. In the TM-AFM dynamics, the tip mass, nonlinear contact forces, damping and intermittent contact can all excite higher modes and higher harmonics into the system motion through the mode coupling mechanism. The higher modes participation in the motion and the necessity of the convergence study are demonstrated even when the driving frequency is well below the first eigenfrequency. The multimodal analysis should be taken, which offers a more general and accurate approach of studying the AFM intermittent contact dynamics. The displacement switching condition allows us to have a more general study on the TM-AFM dynamics. Various subharmonic motions arise due to the intermittent contact. The nonperiodic motion also appears because of relatively large forcing amplitude, or say, large tip-sample interaction. The presence of adhesion is an important factor which may significantly change the system response to a "tip-stuck-to-sample" motion. The response of TM-AFM in the intermittent contact can be so distorted that the assumption of harmonic motion cannot be accurate. When TM-AFM is used to image the sample and measure its related mechanical properties, the extreme care should be taken to interpret the data because of the rich AFM dynamic behaviors and their sensitivity to the initial conditions in the intermittent contact as demonstrated in this study. The "tip-stuck-to-sample" motion, which is insensitive to the initial conditions and the dominance of one mode, should be the type of motion adopted for the inverse problem solving.

\section{Acknowledgments}

The research has been supported by the National Natural Science Foundation of China (NSFC Nos. 11021262 and 11023001) and Chinese Academy of Sciences (Grant no. KJCX2-EW-L03).

\section{References}

[1] G. Binnig, C. Quate, C. Gerber, Atomic force microscope, Physical Review Letters 56 (1986) 930-934.

[2] F.J. Giessibl, Forces and frequency shifts in atomic-resolution dynamic-force microscopy, Physical Review B 56 (1997) $16010-16015$.

[3] B. Gotsmann, C. Seidel, B. Anzykowski, H. Fuchs, Conservative and dissipative tip-sample interaction forces probed with dynamic AFM, Physical Review B 60 (1999) 11051-11061.

[4] H. Hölscher, U.D. Schwarz, Theory of amplitude modulation atomic force microscopy with and without Q-control, International Journal of Non-Linear Mechanics 42 (2007) 608-625.

[5] H. Hölscher, W. Allers, U.D. Schwarz, A. Schwarz, R. Wiesendanger, Determination of tip-sample interaction potentials by dynamic force spectroscopy, Physical Review Letters 83 (1999) 4780-4783.

[6] G. Wang, Y. Zhang, Y. Zhao, G. Yang, Pull-in instability study of carbon nanotube tweezer under the influence of van der Waals force of an archshaped beam under electrostatic loading, Journal of Micromechanics and Microengineering 14 (2004) 1119-1125.

[7] Y. Zhang, Y. Wang, Z. Li, Y. Huang, D. Li, Snap-through and pull-in instabilities of an arch-shaped beam under electrostatic loading, Journal of Microelectromechanical Systems 16 (2007) 684-693.

[8] W.H. Lin, Y.P. Zhao, Nonlinear behavior for nanoscale electrostatic actuators with Casimir force, Chaos, Solitons and Fractals 23 (2005) 1777-1785.

[9] U. Dürig, Relations between interaction force and frequency shift in large-amplitude dynamic force microscopy, Applied Physics Letters 75 (1999) 433-435.

[10] Q. Zhong, D. Inniss, K. Kjoller, V.B. Ellings, Fractured polymer/silica fiber surface studied by tapping mode atomic force microscopy, Surface Science Letters 290 (1993) L688-L692.

[11] H. Li, Y. Chen, L. Dai, Concentrated-mass cantilever enhances multiple harmonics in tapping-mode atomic force microscopy, Applied Physics Letters 92 (2008) 151903.

[12] N.F. Martinez, J.R. Lozano, E.T. Herruzo, F. Garcia, C. Richter, T. Sulzbach, R. Garcia, Bimodal atomic force microscopy imaging of isolated antibodies in air and liquids, Nanotechnology 19 (2008) 384011.

[13] D. Martinez-Martin, E.T. Herruzo, C. Dietz, J. Gomez-Herrero, R. Garcia, Noninvasive protein structural flexibility mapping by bimodal dynamic force microscopy, Physical Review Letters 106 (2011) 198101.

[14] J. Preiner, J. Tang, V. Pastushenko, P. Hinterdorfer, Higher harmonic atomic force microscopy: imaging of biological membrane in liquid, Physical Review Letters 99 (2007) 046102.

[15] J. Melcher, C. Carrasco, X. Xu, J.L. Carrascosa, J. Gómez-Herrero, P.J. de Pablo, Origins of phase contrast in the atomic force microscope in liquids, Proceedings of the National Academy of Sciences of the United States of America 106 (2009) 13655-13660.

[16] O. Sahin, G. Yaralioglu, R. Grow, S.F. Zappe, A. Atalar, C. Quate, O. Solgaard, High-resolution imaging of elastic properties using harmonic cantilevers, Sensors and Actuators A 114 (2004) 183-190.

[17] M. Balantekin, A. Atalar, Enhancing higher harmonics of a tapping cantilever by excitation at a submultiple of its resonance frequency, Physical Review B 71 (2005) 125416.

[18] R.W. Stark, Getting a feeling for the nanoworld, Nature Nanotechnology 2 (2007) 461-462.

[19] U. Dürig, Extracting interaction forces and complementary observables in dynamic probe microscopy, Applied Physics Letters 76 (2000) $1203-1205$.

[20] F.J. Giessibl, A direct method to calculate tip-sample forces from frequency shifts in frequency-modulation atomic force microscopy, Applied Physics Letters 78 (2001) 123-125.

[21] H. Hölscher, Quantitative measurement of tip-sample interactions in amplitude modulation atomic force microscopy, Applied Physics Letters 89 (2006) 123109

[22] M. Lee, W. Jhe, General theory of amplitude-modulation atomic force microscopy, Physical Review Letters 97 (2006) 036104 
[23] N.A. Burham, A.J. Kulik, G. Gremaud, G.A.D. Briggs, Nanosubharmonics: the dynamics of small nonlinear contacts, Physical Review Letters 74 (1995) 5092-5095.

[24] J. de Weger, D. Binks, J. Molenaar, W. van de Water, Generic behavior of grazing impact oscillators, Physical Review Letters 76 (1996) $3951-3954$.

[25] W. van de Water, J. Molenaar, Dynamics of vibrating atomic force microscopy, Nanotechnology 11 (2000) 192-199.

[26] S. Hu, A. Raman, Chaos in atomic force microscopy, Physical Review Letters 96 (2006) 036107.

[27] Y. Zhang, K.D. Murphy, Multi-modal analysis on the intermittent contact dynamics of atomic force microscope, Journal of Sound and Vibration 330 (2011) 5569-5582.

[28] M. Stark, R.W. Stark, W.M. Heckl, R. Guckenberg, Inverting dynamic force microscopy: from signals to time-resolved interaction forces, Proceedings of the National Academy of Sciences of the United States of America 99 (2000) 8473-8478.

[29] R.W. Stark, W.M. Heckl, Fourier transformed atomic force microscopy: tapping mode atomic force microscopy beyond the Hookian approximation, Surface Science 457 (2000) 219-228.

[30] M. Stark, R.W. Stark, W.M. Heckl, R. Guckenberg, Spectroscopy of the anharmonic cantilever oscillations in tapping mode atomic force microscopy, Applied Physics Letters 77 (2000) 3293-3295.

[31] R. Hillenbrand, M. Stark, R. Guckenberger, Higher-harmonics generation in tapping-mode atomic-force microscopy: insights into the tip-sample interaction, Applied Physics Letters 76 (2000) 3478-3480.

[32] R.W. Stark, Spectroscopy of higher harmonics in dynamics atomic force microscopy, Nanotechnology 15 (2004) 347-351.

[33] I.Yu. Sokolov, G.S. Henderson, F.J. Wicks, The contrast mechanism for true atomic resolution by AFM in non-contact mode: quasi-non-contact mode, Surface Science 381 (1997) L558-L562.

[34] P. Attard, J.C. Schulz, M.W. Rutland, Dynamic surface force measurement. I. van der Waals collision, Review of Scientific Instruments 69 (1998) $3852-3866$

[35] K.D. Murphy, Y. Zhang, Vibration and stability of a cracked translating beam, Journal of Sound and Vibration 237 (2000) 319-335.

[36] J. Berg, G.A.D. Briggs, Nonlinear dynamics of intermittent-contact mode atomic force microscopy, Physical Review B 55 (1997) 14899-14908.

[37] A.B. Nordmark, Non-periodic motion caused by grazing incidence in an impact oscillator, Journal of Sound and Vibration 145 (1991) $279-297$.

[38] S.I. Lee, S.W. Howell, A. Raman, R. Reifenberger, Nonlinear dynamics of microcantilevers in tapping mode atomic force microscopy: a comparison between theory and experiment, Physical Review B 66 (2002) 115409.

[39] S.W. Shaw, P.J. Holmes, A periodically forced piecewise linear oscillator, Journal of Sound and Vibration 90 (1983) 129-155.

[40] M. Radmacher, R.W. Tillman, M. Fritz, H.E. Gaub, From molecules to cells: imaging soft samples with atomic force microscope, Science 257 (1992) 1900-1905.

[41] J.H. Hoh, A. Engel, Friction effects on force measurements with an atomic force microscope, Langmuir 9 (1993) 3310-3312.

[42] P. Mazeran, J. Loubet, Force modulation with a scanning force microscope: an analysis, Tribology Letters 3 (1997) 125-132.

[43] R. Garcia, C.J. Gómez, N.F. Martinez, S. Patil, C. Dietz, R. Magerle, Identification of nanoscale dissipation processes by dynamic atomic force microscopy, Physical Review Letters 97 (2006) 016103.

[44] Y. Zhang, K.D. Murphy, Static and dynamic structural modeling analysis of atomic force microscope, in: B. Bhushan (Ed.), Scanning Probe Microscopy in Nanoscience and Nanotechnology, Springer, Berlin, 2010. (Chapter 8).

[45] Y. Zhang, Extracting nanobelt mechanical properties from nanoindentation, Journal of Applied Physics 107 (2010) 123518

[46] S. Jesse, A.P. Baddorf, S.V. Kalinin, Dynamic behavior on piezoresponse force microscopy, Nanotechnology 17 (2006) 1615-1628.

[47] B. Mirman, S.V. Kalinin, Resonance frequency analysis for surface-coupled atomic force microscopy cantilever in ambient and liquid environments, Applied Physics Letters 92 (2008) 083102.

[48] L. Wang, Analytical descriptions of the tapping-mode atomic force microscopy response, Applied Physics Letters 73 (1998) 3781-3783.

[49] D. Xu, K. Ravi-Chandar, K.M. Liechti, On scale dependence in friction: transitional from intimate to monolayer-lubricated contact, Journal of Colloid and Interface Science 318 (2008) 507-519.

[50] Y.P. Zhao, L.S. Wang, T.X. Yu, Mechanics of adhesion in MEMS-a review, Journal of Adhesion Science and Technology 17 (2003) $519-546$.

[51] Y. Zhang, Adhesion map of sphere: effects of curved contact interface and surface interaction outside contact region, Journal of Adhesion Science and Technology 25 (2011) 1453-1464.

[52] K.L. Johnson, Contact Mechanics, Cambridge University Press, Cambridge, 1985.

[53] T.C. Chang, R.R. Craig, Normal modes of uniform beams, Journal of Engineering Mechanics 95 (1969) 1027-1031.

[54] W.H. Press, S.A. Teukolsky, W.T. Vetterling, B.P. Flannery, Numerical Recipes in Fortran, 2nd edition, Cambridge University Press, Cambridge, 1992 (Chapter 16).

[55] O. Sahin, N. Erina, High-resolution and large dynamic range nanomechanical mapping in tapping-mode atomic force microscopy, Nanotechnology 19 (2008) 445717.

[56] R. Garcia, A. San Paulo, Attractive and repulsive tip-sample interaction regimes in tapping-mode atomic force microscopy, Physical Review B 60 (1999) 4961-4967.

[57] J. Tian, Dynamic contact stiffness of adhesive Hertzian contact, Journal of Adhesion Science and Technology 25 (2011) $1087-1094$.

[58] Y.M. Tseytlin, High resonant mass sensor evaluation: an effective method, Review of Scientific Instruments 76 (2005) 115101.

[59] S. Crittenden, A. Raman, R. Reifenberger, Probing attractive forces at the nanoscale using higher-harmonic dynamic force microscopy, Physical Review B 72 (2005) 235422. 